\title{
Design and Assembly of the Pre-Series Quadrupoles for the LHC Insertions
}

\author{
R. Ostojic, J. Lucas, G. Kirby, J. C. Perez, and H. Prin
}

\begin{abstract}
The LHC insertions are equipped with several different types of individually powered superconducting quadrupoles. These units comprise several quadrupole magnets and orbit correctors, and range in length from $5.3 \mathrm{~m}$ to $11.3 \mathrm{~m}$. In spite of the variety of types and interface requirements, the design of the quadrupoles is based on the same principle where two welded half-shells provide the rigidity and alignment of the magnets and serve as a helium pressure vessel. In this paper we present the mechanical design of the LHC insertion quadrupoles, describe the initial experience in the assembly of the pre-series units, and report on the alignment measurements.
\end{abstract}

Index Terms-Insertions, LHC, quadrupoles, superconducting.

\section{INTRODUCTION}

$\mathbf{T}$ HE optics flexibility of the LHC insertions is provided by the individually powered quadrupoles in the dispersion suppressors and matching sections. These units comprise several quadrupole magnets of the MQM or MQY type [1], [2], arranged to give the necessary field strength. In the dispersion suppressors (quadrupole cells Q8 to Q10), the quadrupoles are part of the continuous arc cryostat, operate at $1.9 \mathrm{~K}$, and although independently powered, provide identical cryogenic and powering interfaces to the adjacent main dipoles as in the regular arc cells. Most of the quadrupoles in the matching sections (quadrupole cells Q4 to Q7) are stand-alone units and are cooled in static helium bath at $4.5 \mathrm{~K}$. Although in principle simpler, their cryogenic and powering interfaces are determined by the local conditions of each insertion (e.g., slope of the tunnel, interference with injection lines etc.)

The insertion quadrupoles range in length from $5.35 \mathrm{~m}$ to $11.35 \mathrm{~m}$, and comprise up to seven magnetic elements. The main parameters of the quadrupoles are given in Table I. In total, 82 insertion quadrupoles will be assembled in the LHC Magnet Assembly Facility (MAF) at CERN.

In this paper we present the mechanical design of the LHC insertion quadrupoles, report on the initial experience in the assembly of the pre-series units, and discuss the results of mechanical and alignment measurements.

\section{Mechanical Design of THE InSERTion Quadrupoles}

The conceptual design of the insertion quadrupole is shown in Fig. 1. The central elements of the assembly are the two half-shells which serve for positioning of the various magnetic

Manuscript received October 20, 2003.

The authors are with CERN, Accelerator Technology Division, 1211 Geneva 23, Switzerland (e-mail: Ranko.Ostojic@ cern.ch).

J. Lucas is presently with ELYTT Energy, Portugalete, Spain.

Digital Object Identifier 10.1109/TASC.2004.829047
TABLE I

MAIN PARAMETERS OF THE LHC INSERTION QUADRUPOLES

\begin{tabular}{|c|c|c|c|c|c|}
\hline Position (IR) & Magnets & $\mathrm{T} \quad(\mathrm{K})$ & $\begin{array}{l}\text { Length } \\
(\mathrm{mm})\end{array}$ & $\begin{array}{l}\text { Weight } \\
\text { (ton) }\end{array}$ & $\begin{array}{l}\text { No. } \\
\text { units }\end{array}$ \\
\hline Q7 (IR4) & $\mathrm{MQM}+\mathrm{MCBC}$ & 1.9 & 5345 & 5.8 & 2 \\
\hline Q5, Q6 (IR4) & $\mathrm{MQY}+\mathrm{MCBC}$ & 4.5 & 5345 & 5.8 & 8 \\
\hline \multicolumn{6}{|l|}{ Q4, Q5 (IR6) } \\
\hline Q10, Q8 & $\mathrm{MQML}+\mathrm{MCBC}$ & 1.9 & 6620 & 7.4 & 24 \\
\hline Q5, Q6 (IR1,5) & $\mathrm{MQML}+\mathrm{MCBC}$ & 4.5 & 6620 & 8.2 & 8 \\
\hline Q9 & MQMC+MQM+MCBC & 1.9 & 8020 & 9.3 & 12 \\
\hline Q4 (IR1,5) & $\mathrm{MQY}+3 \mathrm{MCBY}$ & 4.5 & 8020 & 9.3 & 4 \\
\hline $\begin{array}{c}\text { Q7 } \\
(\text { IR } 1,2,5,8)\end{array}$ & $\mathrm{MQM}+\mathrm{MQM}+\mathrm{MCBC}$ & 1.9 & 8995 & 11.10 & 8 \\
\hline Q6 (IR2,8) & $\mathrm{MQM}+\mathrm{MQML}+\mathrm{MCBC}$ & 4.5 & 10400 & 12.3 & 4 \\
\hline Q6 (IR3,7) & $6 \mathrm{MQTL}+\mathrm{MCBC}$ & 4.5 & 10400 & 12.3 & 4 \\
\hline Q4, Q5 (IR2,8) & $2 \mathrm{MQY}+3 \mathrm{MCBY}$ & 4.5 & 11355 & 14.1 & 6 \\
\hline Q5 (IR2,8) & $2 \mathrm{MQM}+3 \mathrm{MCBY}$ & 4.5 & 11355 & 14.1 & 2 \\
\hline
\end{tabular}

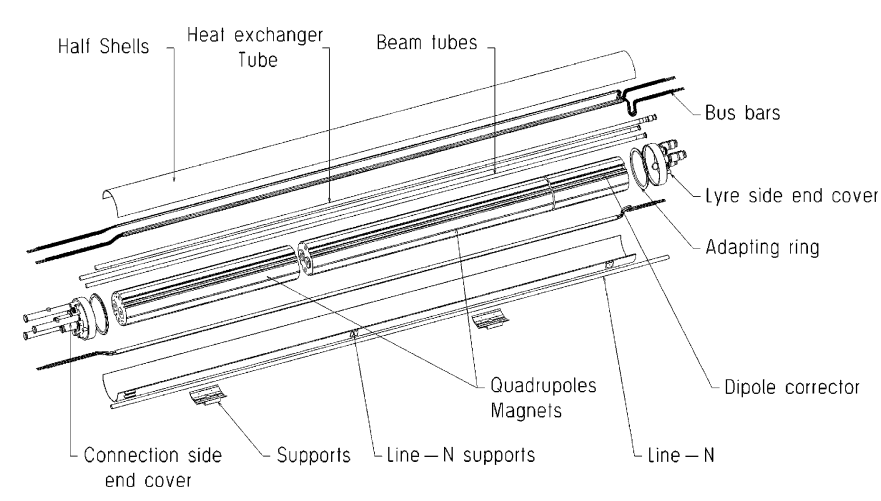

Fig. 1. Isometric view of the insertion quadrupole.

elements (quadrupoles and dipole orbit correctors), provide the rigidity for their alignment and serve as a helium pressure vessel. The vessel is closed with two end covers, which also support the elements required for interconnecting the string of LHC cryo-magnets. In particular, the main $13 \mathrm{kA}$ electrical bus work and $1.9 \mathrm{~K}$ helium lines are guided by the end covers, and the beam position monitors (BPM) and beam vacuum interconnects are precisely positioned with end covers as reference. The quadrupole is supported in the vacuum vessel on two supports (except for the $10.4 \mathrm{~m}$ and $11.35 \mathrm{~m}$ long quadrupoles, which have three supports). The thermal shield and other cryostat elements are similar to the LHC main quadrupole [3]. The line-N, which carries the 600 A bus work for the corrector circuits in the LHC arc, also houses the $6 \mathrm{kA}$ cables for powering the insertion quadrupoles.

The design of the insertion quadrupole was validated on several models and on a $3 \mathrm{~m}$ prototype. In the following, the main design features are illustrated using examples from the prototype and pre-series quadrupole assembly. 


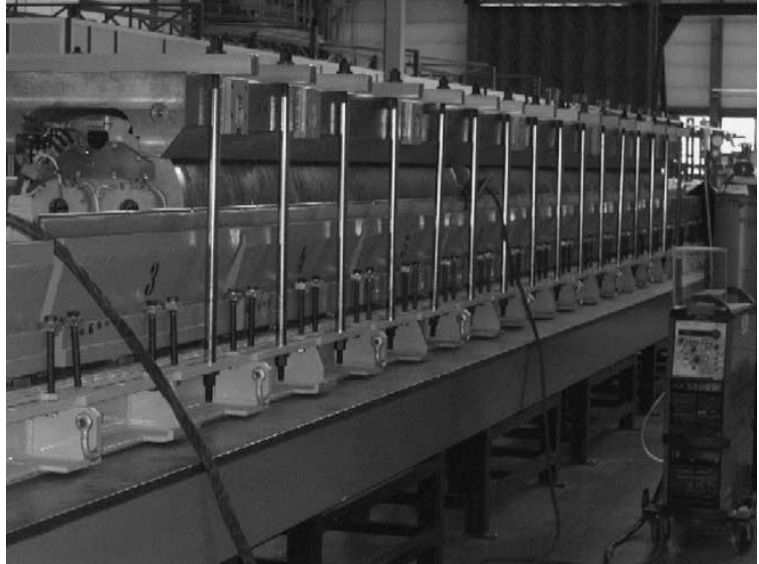

Fig. 2. Alignment of the pre-series quadrupole. The magnets are placed in the half shell in the welding cradles. The alignment cradles are positioned on the yoke flats. Tie bars distribute the force between the alignment cradles and the assembly table.

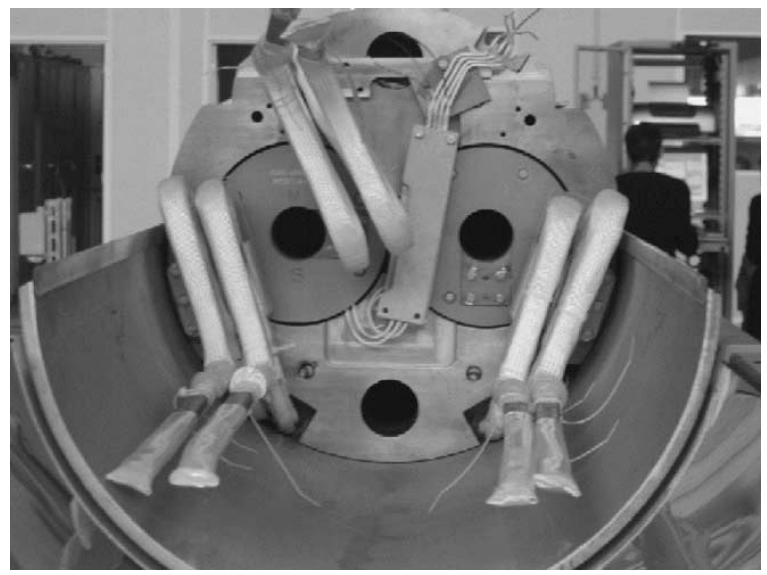

Fig. 3. Non-connection side of the pre-series quadrupole showing the expansion loops of the three sets of $13 \mathrm{kA}$ bus bars.

\section{A. Magnet Alignment}

One of the most delicate steps in the assembly of the quadrupoles is the alignment of the magnets, and its preservation throughout the process. Precision alignment cradles are used, in the first instance to individually align the magnets, which are not aligned during yoke assembly [1], [2]. The yoke laminations have alignment flats designed as contact surfaces for the tooling, and also as references for control. After compressing the cradles, the alignment of individual magnets is completed by tack welding the alignment keys to the laminations.

Subsequently, the individually aligned magnets are placed in the half-shell. They are aligned relative to each other using the cradles which span the full length of the assembly. The operation is completed with tack welding of the alignment keys to the chamfer of the half-shell. Initially, this operation was to be performed in the $17 \mathrm{~m}$ welding press in the MAF. During assembly of the prototype, it was found that the force needed was small and that better control and quicker assembly could be obtained using tie bars, as shown in Fig. 2.

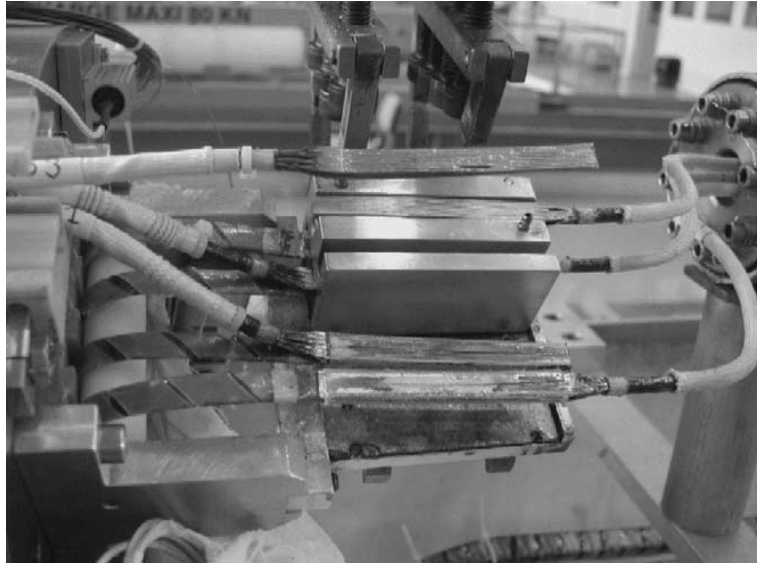

Fig. 4. Jointing of the $6 \mathrm{kA}$ bus bars from line-N (right) with the internal $6 \mathrm{kA}$ bus bar (left) and the flat lead cables of the MQM magnet (center).

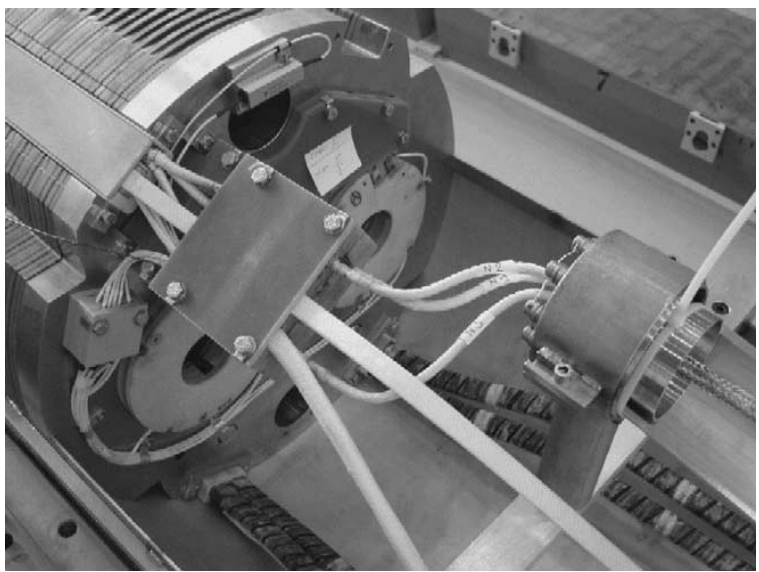

Fig. 5. Connection end of the quadrupole after completion of the $6 \mathrm{kA}$ splices. The helium tight feed-through for the three $6 \mathrm{kA}$ round wires to line- $\mathrm{N}$ is shown on the right. The $13 \mathrm{kA}$ main quadrupole bus bars, bent in situ to their final positions, are in the bottom of the assembly.

\section{B. Electrical Connections}

Several electrical circuits and their instrumentation are installed during assembly. In the first place, the $13 \mathrm{kA}$ main LHC dipole and quadrupole bus work, although not used for powering the insertion quadrupoles, must be installed to provide continuity of the arc circuits. Although the design of the bus bars follows closely that of the main magnets [4], there are a number of differences, particularly in the position of the expansion loops, shown in Fig. 3. Furthermore, as the main quadrupole bus bars are pulled through after the magnets are aligned in the half-shell, their leads ends have to be bent in situ to their final position.

The insertion quadrupoles are powered with three $6 \mathrm{kA}$ round cables [5] routed through line-N (external to the helium vessel), which allow independent powering of the two apertures. In those quadrupoles which comprise two magnets, Table I, the apertures are powered in series, and the bus bars connecting the magnets are also $6 \mathrm{kA}$ round cables. Several splices between the $6 \mathrm{kA}$ round cables and the magnet Rutherford-type cable are therefore required. The solution developed is based on preparing the extremities of the round cable as two flat layers, and soldering the magnet leads in between. The connections on the lead side of the quadrupole, shown in Fig. 4, are particularly challenging due to large number of splices and tight space. The lead side of 


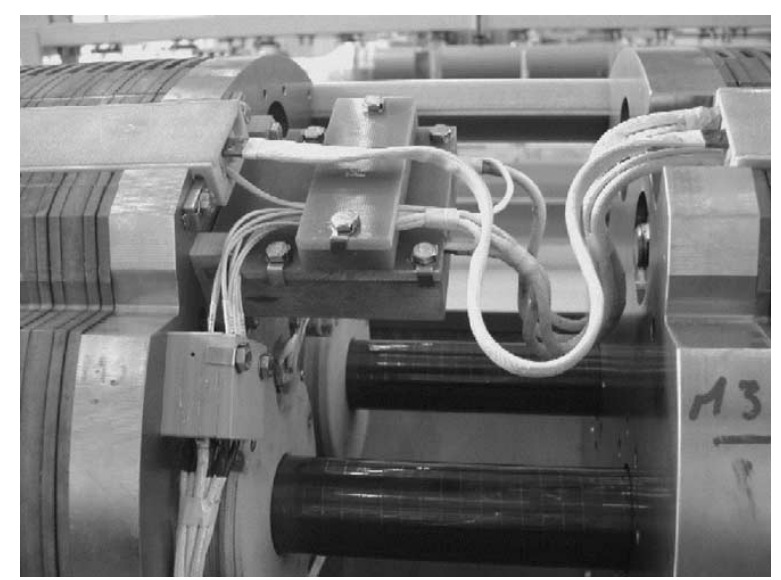

Fig. 6. Interconnection between the two MQM magnets showing the $6 \mathrm{kA}$ connection box and instrumentation wire block (left), and the expansion loop for the $1 \mathrm{kA}$ dipole corrector bus bar (right).

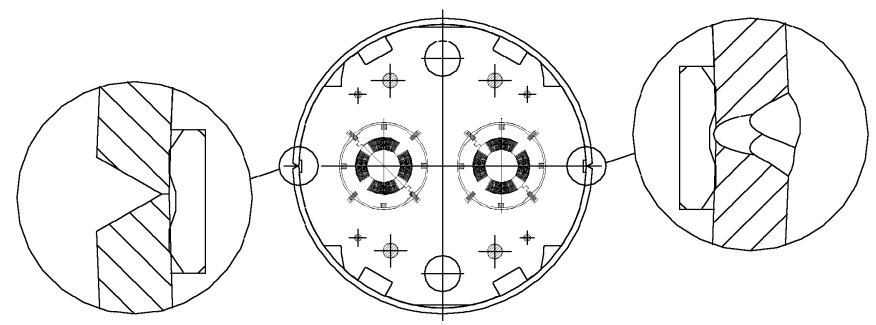

Fig. 7. Geometry of the longitudinal weld before (left) and after (right) welding.

the pre-series quadrupole after completion of the connections is shown in Fig. 5, where the helium tight feed-through separating the helium volumes of the quadrupole and line-N is also shown. The same technique is used for interconnecting the $6 \mathrm{kA}$ bus bars with the magnet leads of the second magnet, Fig. 6.

\section{Longitudinal Weld}

Considerable effort has been spent for the development and qualification of welds, in particular of the longitudinal weld. The design finally adopted is a three pass MIG weld, shown in Fig. 7, in a cavity formed by the shell chamfers and alignment key having the function of a backing strip. Before welding, the root faces of the two half-shells are in contact. Their inner circumference is larger than that of the yoke by $3 \mathrm{~mm}$, corresponding to the weld shrinkage. After welding, the shells and the laminations are in contact around the circumference, nominally with zero interference. The longitudinal weld is performed in the $17 \mathrm{~m}$ welding press, Fig. 8, revised for the production of insertion quadrupoles.

\section{End Covers}

The end covers used for closing the quadrupole helium vessel are identical to those of the LHC main dipole. However, many of their features had to be modified so that quadrupole-specific cryogenic tubing and other elements can be mounted. In general, the tolerances for positioning of the tubes are very tight, and special tools and procedures are necessary. In Fig. 9, the set-up for welding of the cryogenic tubes is shown. Experience with the first end covers showed that the average offset of the extremities

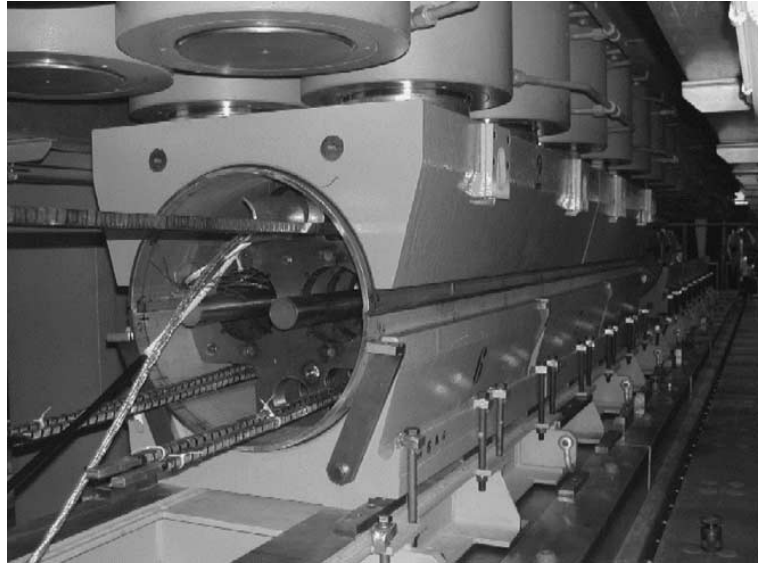

Fig. 8. 3-m quadrupole prototype in place for longitudinal welding.

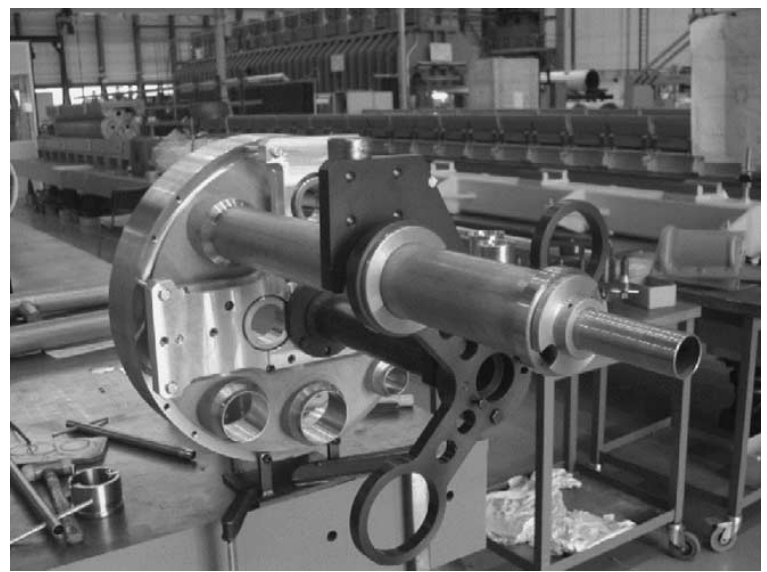

Fig. 9. Positioning and welding of the cryogenic tubes on the end domes.

of the tubes ( $680 \mathrm{~mm}$ from the end cover) is $\pm 0.30 \mathrm{~mm}$, within the tolerance of $\pm 0.50 \mathrm{~mm}$.

One of the most critical assembly stages is the welding of the end covers. Prior to this operation, the shells are cut to precise length and machined for mounting of the adapting rings, which compensate the difference in outer diameters of the shell and end covers. Due to the importance of the final positions of the cryogenic tubes, the final positioning of the end covers is controlled by a laser tracker. Before the final orbital weld, the end covers are tack welded and their position adjusted, as shown in Fig. 10. The final step in completing the quadrupole extremities is the mounting of the BPM supports, Fig. 11, also precisely controlled with a laser tracker.

\section{Alignment Measurements}

The experience gained so far in assembly of the insertion quadrupoles is based on the assembly of the prototype, prealignment of five MQM magnets, and assembly of the $8 \mathrm{~m}$ long Q9 quadrupole (MQMC+MQM+MCBC magnets).

Before pre-alignment, the maximum values of the transverse offset and lamination twist of the magnets were $0.33 \mathrm{~mm}$ and $1.31 \mathrm{mrad}$. After pre-alignment, the maximum values were $0.21 \mathrm{~mm}$ and $0.51 \mathrm{mrad}$, respectively, confirming the effectiveness of the operation.

The alignment of the Q9 quadrupole is shown in Fig. 12, where a comparison is shown of the alignment in the horizontal 


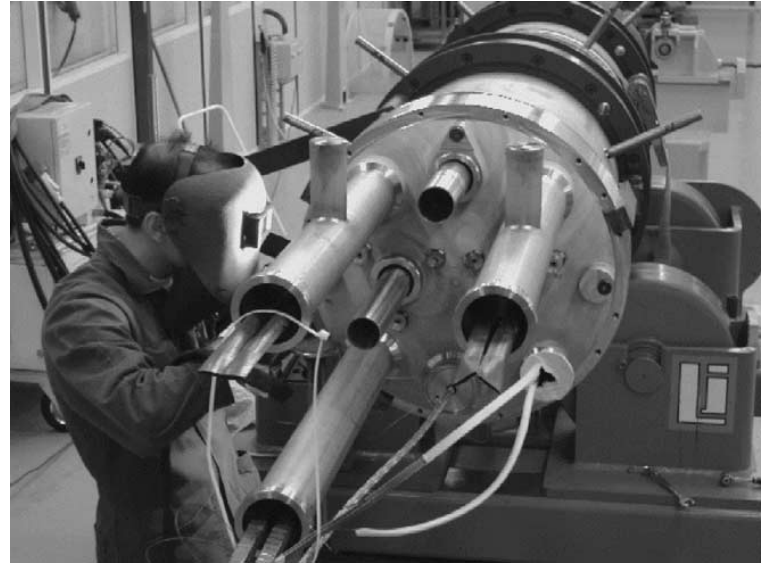

Fig. 10. Positioning of the end covers before final orbital welding.

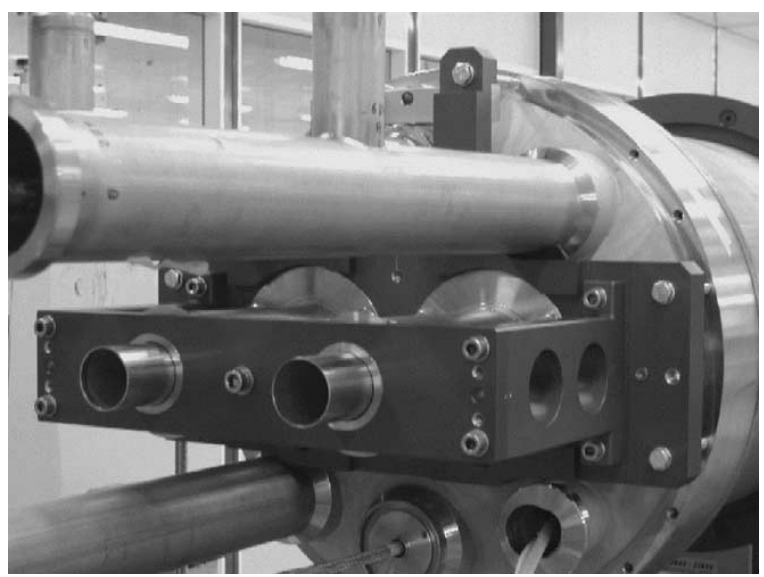

Fig. 11. Positioning of the BPM bodies on the connection side of the pre-series quadrupole.

and vertical planes before and after welding of the half-shells. The measurements before welding are based on the position of the yoke alignment flats, while after welding, a measurement was made by tracking an auto-centering mole through the beam tubes. Both measurements are reported in the reference system related to the magnet center.

Contrary to the measurements of the yoke flats, the beam tube measurements give direct insight into the geometry of the magnet apertures. However, these measurements also include the tolerances of the bore tube (straightness and wall thickness) and its position within the aperture. Furthermore, before welding of the BPM supports, the beam tubes are free to move by as much as $1 \mathrm{~mm}$ in the ends. Also, the natural sag of the magnet in the vertical plane is of the order of $0.2 \mathrm{~mm}$. In spite of all these effects, the two measurements coincide remarkably well, particularly in the center of the quadrupole.

The measurements of the end covers on the prototype showed that they are mounted within $0.16 \mathrm{~mm}$ of the magnet center, while the position of the beam tubes are on average off-centered by $0.22 \mathrm{~mm}$. The cryogenic tubes are within $0.60 \mathrm{~mm}$ of their theoretical position. All values reported above are within the tolerances required for the geometry of the insertion quadrupoles. Although the tolerances are very tight, the initial experience demonstrates that the tooling and procedures are appropriate for this demanding work.
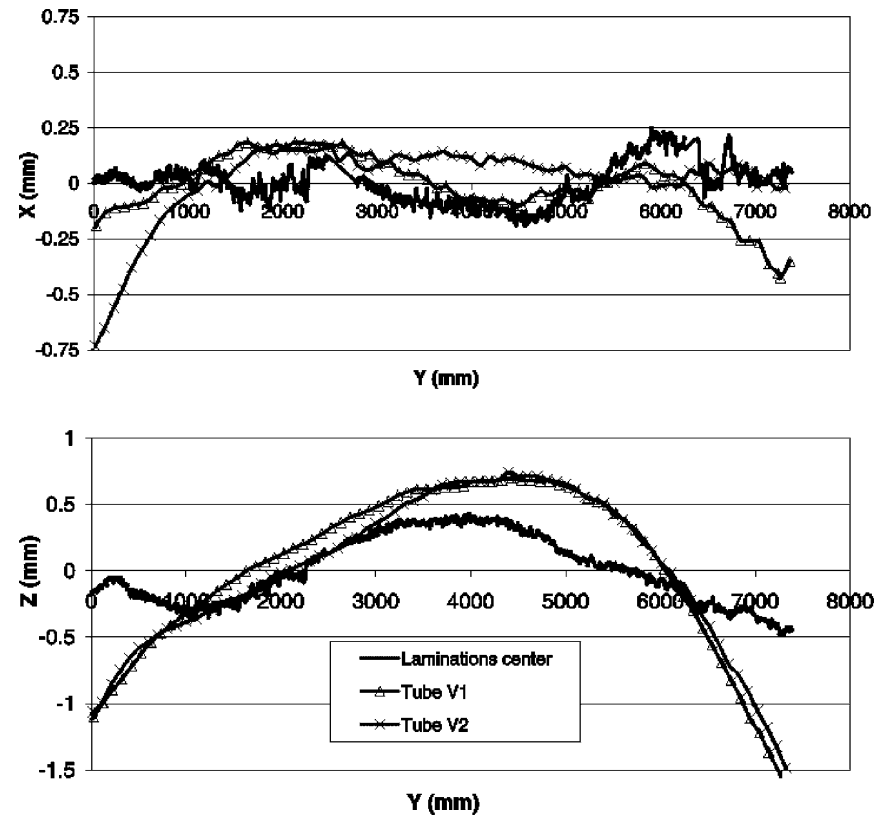

Fig. 12. Comparison of the alignment of the Q9 pre-series quadrupole in the horizontal (top) and vertical (bottom) planes before and after welding of the half shells. Measurements of the yoke laminations (line) and beam tubes V1 and V2 (symbols) were made with a laser tracker.

\section{CONCLUSIONS}

Several types of quadrupoles are required for the LHC insertions, ranging in length from $5.3 \mathrm{~m}$ to $11.3 \mathrm{~m}$. They are all based on the same construction principle, where the welded half-shells provide the rigidity for aligning the magnets and serve as the helium pressure vessel. The tooling and procedures for the quadrupole assembly, including electrical connections, longitudinal welds and vessel closure, and the associated test procedures, have been developed and verified on models and a prototype assembly. The measurements of the pre-series quadrupole have shown that very strict requirements for magnet alignment can be met.

\section{ACKNOWLEDGMENT}

The authors would like to thank the technicians in the LHC Magnet Assembly Facility for their competence, commitment and hard work in the assembly of the insertion magnets.

\section{REFERENCES}

[1] R. Ostojic, N. C. Lasheras, J. Lucas, W. V. Delsolaro, and D. Landgrebe, "Construction and Qualification of the Pre-Series MQM-Type Superconducting Quadrupoles for the LHC Insertions,", this Conference.

[2] G. Kirby, R. Ostojic, R. Burgmer, M. Jordan, D. Krichel, and P. Schmidt, Fabrication of the Pre-Series Wide Aperture Superconducting Quadrupoles for the LHC Insertions, vol. this Conference.

[3] J.-B. Bergot et al., "A modular design for the 56 variants of the short straight section in the arcs of the Large Hadron Collider (LHC)," in Proc. EPAC 2000, Vienna, June 2000, pp. 2157-2160.

[4] L. Belova et al., "Design and manufacture of the superconducting bus-bars for the LHC main magnets," IEEE Trans. Appl. Supercond., vol. 12, no. 1, pp. 1305-1309, March 2002.

[5] M. Calvi, R. Herzog, M. Pelegrin-Carcelen, and F. Sonnemann, "Quench propagation in the superconducting $6 \mathrm{kA}$ flexible busbars of the LHC," Adv. Cry. Engr., vol. 47, pp. 583-590, 2002. 